\title{
Cooperativismo de segundo grado y adopción de las TIC
}

\section{Adoración Mozas Moral Enrique Bernal Jurado Domingo Fernández Uclés Miguel Jesús Medina Viruel Raquel Puentes Poyatos}

RESUMEN: el presente trabajo de investigación se centra en el estudio de los factores determinantes para la adopción de las herramientas de Tecnologías de la Información y Comunicación con objetivos comerciales en las cooperativas oleícolas de segundo grado en España. El sector oleícola español lidera la producción mundial de aceite de oliva, sin embargo, cuenta con importantes problemas comerciales que ponen en riesgo la rentabilidad de los productores. Éste es un problema históricamente ligado a este sector. En este sentido el cooperativismo de segundo grado salva una de las trabas para su avance comercial frecuentemente señalada por la literatura sobre el sector oleícola, como es la falta de concentración e integración de la oferta. Con el fin de alcanzar el objetivo establecido en este trabajo de investigación se ha hecho uso de la técnica metodológica fuzzy set Qualitative Comparative Analysis (fsQCA). Los resultados extraídos señalan que el grado de innovación tecnológica se ve favorecido por la intensidad de integración cooperativa, la formación en TIC de los empleados, la importancia comercial del sector exterior, la oferta de productos ecológicos dentro de la gama de productos de la cooperativa y el grado de compromiso de la cooperativa respecto a las acciones de Responsabilidad Social Corporativa (RSC).

PALABRAS CLAVE: cooperativismo segundo grado; aceite de oliva; innovación; formación; Responsabilidad Social Corporativa (RSC); integración; internacionalización; producción ecológica;

CÓDIGOS JEL: M15; 032; P13

Cómo citar este artículo/How to cite this article: MOZAS, A., BERNAL, E., FERNÁNDEZ, D., MEDINA, M. J. \& PUENTES, R. (2020): “Cooperativismo de segundo grado y adopción de las TIC", CIRIEC-España, Revista de Economía Pública, Social y Cooperativa, 100, 67-85. D0I: 10.7203/CIRIEC-E.100.17712.

Correspondencia: Adoración Mozas Moral,Universidad de Jaén, amozas@ujaen.es, ORCID: 0000-00019858-2392; Enrique Bernal Jurado, Universidad de Jaén, ebernal@ujaen.es, ORCID: 0000-0001-82412866; Domingo Fernández Uclés, Universidad de Jaén, dfucles@ujaen.es. ORCID: 0000-0001-7335-0296; Miguel Jesús Medina Viruel, Universidad de Córdoba, mjmedina@uco.es, ORCID : 0000-0001-8448-3393; Raquel Puentes Poyatos, Universidad de Jaén, rpuentes@ujaen.es, ORCID: 0000-0001-7042-2880. 


\section{Expanded abstract}

\section{Second degree cooperativism and ICT adoption}

Scientific literature points to innovation and investment in R\&D as the main engines of economic growth, as well as job creation in a given country or region (Feldman, 1993 and Mendez 1998). Schumpeter (1934) already predicted that technological change would be decisive, assuming that companies that were not capable of adapting to innovations would not survive in the market. In this sense, Porter (1985 and 1990) indicated what competitiveness was: "competitive advantage derives from the way in which companies organize and carry out discrete activities.... To gain competitive advantage over its rivals, a company must offer a service at a value comparable to that of the competitor, but carry out the activities more efficiently than its competitors (lower cost) or carry out the activities in a peculiar way that creates greater value for the buyer and allows for an overpricing (differentiation)". Undoubtedly, the correct use and management of ICTs constitutes a differentiation (Fernández, Bernal, Mozas and Medina, 2019; Moreno and Sempere, 2019).

ICTs, therefore, have emerged as necessary elements for achieving economic development. This is such an accepted fact that the European Union indicates that the development of ICTs is vital for Europe's competitiveness in today's increasingly digitalized world economy. During the 2014-2020 funding period, the European Regional Development Fund (ERDF) set aside more than 20,000 million euros for investment in ICTs and the European Commission established the Digital Europe 2021-2027 program, which aims to support the digital transformation of the European economy and society and, in this way, transfer its benefits to European citizens and companies (Regulation of the European Parliament and Council establishing the Digital Europe program for the period 2021-2027). Within this digitalization process, made more urgent by Covid-19, cooperatives seem the best way to channel the collaborative economy, under the modality of working on a digital platform (Arrieta, 2019).

In this paper we focus our analysis on one family of the Social Economy: agricultural cooperatives. Specifically, we will go into the analysis of the Spanish olive cooperative movement for several reasons: a) Spain is the world's leading producer of olive oil and more than $70 \%$ of it is produced by cooperatives (Cooperativas Agroalimentarias de España, 2017) and the Consejo Económico y Social de la Provincia de Jaén, 2019). According to the data provided by the International Olive 0il Council (2019 a and b) for the 2018-19 campaign, Spain has produced more than $51 \%$ of the world's olive oils and the new plantations predict a constant increase; b) there are $11,316,000$ hectares in the world that are exclusively dedicated to the cultivation of 1500 million olive trees ( $72 \%$ are mountain and hill), located mainly in the Mediterranean basin (79\%), which gives them an enormous environmental value (at the same time that they serve to reduce erosion and constitute a $\mathrm{CO} 2$ sink) which leads them to be considered by many 
researchers as public goods (Mozas, 2019b); c) annually, an increase in the world's olive grove surface area is observed, which represents 1\% per year (between 34 and 45 million seedlings, which means 154,000 new hectares). The importance of the olive grove is spreading worldwide, since there are already 56 countries in which the olive grove is cultivated and 169 in which its products are consumed (Vilar and Cárdenas, 2016) and these figures are increasing year after year (Mozas, 2019b); d) on the other hand, the production of olive oil with respect to the total of oils and fats produced in the world represents barely $2 \%$. However, consumption has been growing at between 2 and $4 \%$ annually, which is why companies are interested in this product. In fact, the turnover of the sector has experienced a growing increase in recent years, ranging from 9,000 to 13,600 million euros per year and employing more than 30 million people (Vilar and Cárdenas, 2016). All these reasons make it a strategic sector worldwide and for Spain in particular.

The main objective we have set ourselves is to verify which are the structural, organizational and commercial factors that favor technological innovation in the second degree olive oil cooperatives in Spain.

From the theoretical framework that has been analyzed, the following work proposals have been derived:

Proposition 1. Training employees in ICTs favors innovation in the organization.

Proposition 2. The application of measures aimed at commitment to CSR favors innovation in the organization.

Proposition 3. The degree of internationalization, measured by the percentage of exports, favors innovation in the organization.

Proposition 4. The commitment to organic production favors innovation in the organization.

Proposition 5. The degree of business integration, measured through the number of member cooperatives, favors innovation in the organization.

\section{Population and method}

The organizations analyzed in this study have a total of 449 companies (444 cooperatives: 1 SAT; 3 SA; 1 cooperative group), most of them first degree cooperatives, integrating more than 165,000 individual members with a turnover of more than 2 billion euros and employing more than 2,500 people. These organizations are integrated around 35 second degree cooperative societies dedicated to the marketing of olive oil in Spain and constitute our population under study. In order to collect part of the data of this study a survey has been carried out on the population under study, but others were requested from Agro-Food Cooperatives in Spain within the framework of the work of the Socioeconomic Observatory of Spanish Agro-Food Cooperativism (OSCAE).

The methodological technique used in this study was Qualitative Comparative Analysis (QCA), using the fuzzy set approach (fsQCA) in order to establish technological and organizational variables that together are associated with a higher level of efficiency. The QCA technique, based on Boolean algebra, uses a verbal, conceptual and mathematical language that configures it as a qualitative and quantitative approach, combining the main advantages of 
both (Ragin, 1987). Thus, applying QCA it is possible to systematically analyze a set of cases in order to determine causal patterns in the form of need and sufficiency relationships between a set of conditions and an outcome (Scheider \& Wagemann, 2010). This method has the advantage over a regression technique of establishing relationships between subsets of variables to explain relationships. In addition, this technique allows working with medium-size samples not large enough to apply traditional quantitative methods (Ragin et al., 2003; Ragin and Rihoux, 2009).

Specifically, in this study the fuzzy set variant (fsQCA) has been used, since it resolves one of the main drawbacks and criticisms of the initial method, called csQCA, which is its strictly dichotomous approach (Sehring et al., 2013).

The dependent variable is the degree of technological innovation. This variable has been created by considering the different ICTs used by the company in its business performance. Thus, a count has been made of the number of technologies used by the company, considering the following: intranet, website, presence in social networks, e-shop, presence in electronic markets and apps. For their part, the causal variables used have been: training in ICT of human resources (staff and top management), the degree of commitment to Corporate Social Responsibility, the export nature, the sale of ecological supply, and business integration.

The results obtained in this study allow us to verify, by means of the fuzzy set Qualitative Comparative Analysis (fsQCA) technique, that the immersion of the companies in ICTs is favored by the following factors: the intensity of cooperative integration, measured by the number of first degree cooperatives or other entities that make up the second degree cooperative; the ICT training of the employees; the export character of the cooperative, the offer of ecological products and the degree of commitment of the cooperatives to Corporate Social Responsibility (CSR) actions.

The original value of this study is the direct link between economic development and innovation and investment in Information and Communication Technologies (ICT). Furthermore, the conclusion we draw is that far from considering Corporate Social Responsibility as a burden for companies and institutions, it should be analyzed and integrated into organizations as an opportunity. The relationship between these variables will improve not only the competitive position of the company, but also the situation of its environment. 


\section{Introducción}

La literatura científica apunta a la innovación y la inversión en I+D como los principales motores del crecimiento económico, así como de la creación de empleo de un país o región (Feldman, 1993 y Méndez 1998). Schumpeter (1934) ya auguraba que el cambio tecnológico sería decisivo, al asumir que, las empresas que no fuesen capaces de adaptarse a las innovaciones no sobrevivirán en el mercado. En este sentido, Porter (1985 y 1990) ya indicaba lo que era la competitividad: "la ventaja competitiva se deriva de la forma en que las empresas organizan y llevan a cabo actividades discretas... Para conseguir ventaja competitiva respecto a sus rivales, una empresa ha de ofrecer un servicio a un valor comparable al competidor, pero llevar a cabo las actividades de forma más eficiente que sus competidores (coste inferior) o realizar las actividades de una forma peculiar que cree mayor valor para el comprador y permita obtener un sobreprecio (diferenciación)". Sin duda, el uso y la gestión correcta de las TIC constituye una diferenciación (Fernández, Bernal, Mozas y Medina, 2019; Moreno y Sempere, 2019).

Las TIC, por tanto, se han erigido como elementos necesarios para conseguir el desarrollo económico. Es un hecho tan aceptado, que la Unión Europea indica que el desarrollo de las TIC es vital para la competitividad de Europa en una economía mundial actual cada vez más digitalizada. Durante el periodo de financiación 2014-2020, el Fondo Europeo de Desarrollo Regional (FEDER) reservó más de 20.000 millones de euros para inversiones en TIC y la Comisión Europea estableció el programa Europa Digital 2021-2027, que persigue apoyar la transformación digital de la economía y la sociedad europea y, de esta forma, trasladar sus beneficios a los ciudadanos y empresas europeas (Reglamento del Parlamento Europeo y del Consejo por el que se establece el programa Europa Digital para el período 2021-2027). En este proceso de digitalización, acuciado por la Covid-19, las cooperativas constituyen la mejor forma de encauzar la economía colaborativa, bajo la modalidad de trabajo en plataforma digital (Arrieta, 2019).

En este trabajo centramos nuestro análisis en una familia de la Economía Social: el cooperativismo agrario. Concretamente, nos adentraremos en el análisis del cooperativismo oleícola español por varias razones: a) España es el principal productor de aceites de oliva del mundo y más del 70\% se produce en cooperativas (Cooperativas Agroalimentarias de España, 2017) y Consejo Económico y Social de la Provincia de Jaén, 2019). Según los datos aportados por el Consejo Oleícola Internacional (2019 a y b) para la campaña 2018-19, España ha producido más del $51 \%$ de los aceites de oliva del mundo y las nuevas plantaciones auguran un incremento constante; b) en el mundo existen 11.316 .000 hectáreas que se ocupan exclusivamente del cultivo de 1.500 millones de olivos ( $72 \%$ son de montaña y colina), ubicados principalmente en la cuenca del Mediterráneo (79\%), lo que les otorga un valor medioambiental enorme (a la vez que sirven para reducir las erosiones y constituyen un sumidero de C02) lo que les lleva a ser consideramos por muchos investigadores como bienes públicos (Mozas, 2019b); c) anualmente, se constata un incremento de la superficie mundial del olivar que supone el $1 \%$ por año (entre 34 y 45 millones de plantones, lo que supone 154.000 hectáreas nuevas). La importancia del olivar se está extendiendo a nivel mundial, puesto que ya son 56 los países en los que se cultiva el olivar y 169 en los que se consume (Vilar y Cárdenas, 2016) y esas cifras 
van en aumento año tras año (Mozas, 2019b); d) por otra parte, la producción de los aceites de oliva respecto al total de aceites y grasas producidas en el mundo representa apenas el $2 \%$. Sin embargo, el consumo ha ido creciendo entre un 2 y un $4 \%$ anualmente, razón por la que las empresas se interesan por este producto. De hecho, la cifra de negocios del sector ha experimentado un creciente aumento en los últimos años, oscilando entre los 9.000 y 13.600 millones de euros por ejercicio y dando empleo a más de 30 millones de personas (Vilar y Cárdenas, 2016). Todas estas razones lo convierten en un sector estratégico a nivel mundial y para España, en particular.

El objetivo de este trabajo es analizar qué factores influyen en la generación de una mayor innovación medida a través del uso de las TIC en las empresas cooperativas oleícolas de segundo grado en España. Así, mediante el uso de la técnica fuzzy set Quality Coparative Analysis (fsQCA) se estudiará la influencia de variables señaladas en la reciente literatura como determinantes para la adopción de innovaciones tecnológicas en la empresa. Después de esta introducción, donde justificamos el problema de investigación y se definen los objetivos, en el segundo epígrafe nos ocupamos de revisar el marco teórico en la literatura reciente y establecemos las proposiciones del estudio. El apartado tercero, se centra en la metodología seguida y la población objeto de estudio, para continuar en el cuarto apartado con la exposición de los resultados alcanzados. El artículo termina con el planteamiento de las principales conclusiones a las que llegamos en el estudio.

\section{Marco teórico e hipótesis de investigación}

El capital humano tiene un papel determinante en la economía del conocimiento (López et al., 2006), más aún en entornos altamente competitivos, como es el actual mercado del aceite de oliva. La formación de los recursos humanos es un factor determinante para tener una actitud innovadora (Hollenstein, 2004), siendo necesaria una formación continua del personal para adquirir conocimientos útiles y actualizados al entorno en el que actúa la empresa (García et al., 2008), como la adquisición de conocimientos tecnológicos (Medina et al., 2019).

Así, para la obtención de una actitud innovadora en la empresa es esencial contar con directivos que tengan una alta formación y actualizada a las necesidades imperantes en el mercado (Levie y Autio, 2011). Esto hará que se estimule en la organización la formación de los trabajadores y, por tanto, la innovación dentro de ella (Fernández et al., 2019). Con base en estos argumentos teóricos planteamos la siguiente proposición:

\section{Proposición 1. La formación de los empleados en TIC favorece la inno- vación en la organización.}

La responsabilidad social corporativa está de moda por el desarrollo y extensión de los Objetivos de Desarrollo Sostenible (Mozas, 2019a; Villaescusa, 2019). Asimismo, es preciso trans- 
mitir y asegurar la educación cooperativa en el contexto actual (Eba y Gonzalo, 2019). La innovación y la RSC comparten un estrecho vínculo bidireccional (MacGregor y Fontrodona, 2008). La apuesta por la RSC aumenta la denominada innovación social de la organización, lo que conduce a su vez a una mayor RSC, originando un círculo virtuoso que provoca una retroalimentación continua de estas dos variables (Rexhepi et al., 2013). La apuesta por acciones responsables, tanto sociales, económicas o medioambientales mejora la capacidad organizacional hacia actuaciones sociales efectivas, también relacionadas con la innovación, en la búsqueda de las mejores soluciones en sistemas tecnológicos de información y comunicación (Cegarra et al., 2016). Aunque la literatura científica señala una cierta relación entre RSC e innovación, las aportaciones establecidas son principalmente de carácter conceptual, demandándose más evidencias que determinen la relación de ambas variables (Ratajczak y Szutowski, 2016). Ello hace necesario estudiar la relación entre innovación y responsabilidad, esperándose una vinculación positiva, que conducirá a un mejor desempeño organizacional (Pedersen et al., 2018). Con base en estos argumentos, se plantea la siguiente proposición:

\section{Proposición 2. La aplicación de medidas orientadas al compromiso con la RSC favorece la innovación en la organización.}

En la actualidad vivimos inmersos en un mercado global cada vez más competitivo en el que las empresas deben actuar. En este contexto, las innovaciones tecnológicas se convierten en herramientas indispensables para actuar en el mercado internacional. Por ejemplo, la disposición de un sitio web de alta calidad y disponible en diferentes idiomas es una manera de bajo coste de internacionalizar a la empresa (Manyinka y Lund, 2016) y acceder a un mayor número de potenciables clientes (Kim y Jee, 2007). Asimismo, los sitios web permiten a empresas de pequeño tamaño acceder a las exportaciones de manera más eficaz (Qurratu'Aini y Hapsari, 2019). Esta relación también existe en sentido contrario, es decir, el conocimiento tecnológico y el acceso a innovaciones comerciales puede ser incrementado tanto a través de un desarrollo interno como a través de las relaciones de la empresa con otras organizaciones del exterior (Sher y Yang, 2005; Fierro et al., 2013). Además, la exportación contribuye al desarrollo e innovación de la organización y de su sector (Prieto y Guzmán, 2019). Con base en esta argumentación es posible plantear la siguiente proposición:

\section{Proposición 3. El grado de internacionalización, medido a través del porcentaje de exportación, favorece la innovación en la organización.}

El sector de producción ecológica se encuentra, en la actualidad, en continuo crecimiento. No obstante, existen importantes barreras al incremento de la demanda de este tipo de productos, como la desinformación de los consumidores, la falta de puntos de venta y el alto diferencial entre los productos ecológicos y sus homólogos convencionales, siendo estos problemas aún más pronunciados en el sector del aceite de oliva (Vega et al., 2018). Esto está llevando al consumidor al uso creciente de los medios digitales como canal alternativo de compra (Fernández et al., 2019). Además, los consumidores de productos ecológicos tienden a ser más activos 
en Internet, en parte, por su mayor necesidad de información (Lee y Yun, 2015), por lo que estos consumidores harán un mayor uso de sitios web que les ofrezcan información rica y que estén bien diseñados (Bernal, Mozas, Fernández y Medina, 2017). Esta situación llevará a las empresas a realizar una apuesta decidida por la innovación, especialmente comercial. Por su parte, las Naciones Unidas defiende la necesidad de innovación tecnológica como herramienta para la consecución de los Objetivos de Desarrollo Sostenible (Mozas, 2019b). Con base en la argumentación anterior podemos plantear la siguiente proposición:

\section{Proposición 4. La apuesta por la producción ecológica favorece la inno- vación en la organización.}

La estrategia de crecimiento empresarial ya la recogía Ansoff (1976) cuando la identificaba con la estrategia que hace referencia al incremento de tamaño de la organización, pero encaminada al fortalecimiento de la misma actividad que venía desarrollando. El crecimiento es interpretado como un signo de salud, vitalidad y fortaleza y, además en entornos dinámicos y competitivos, las empresas tienen que crecer, aunque sólo sea para mantener su posición competitiva (Guerras y Navas, 2015). La relación entre el tamaño de la organización y su propensión a la innovación es un tema ampliamente estudiado. Así, el tamaño es uno de los principales factores que afectan a la actitud innovadora de las empresas (Rothwell, 1983), siendo las grandes empresas más propensas a las innovaciones tecnológicas (Tether, 2005).

Entre los condicionantes que dificultan la innovación a las pequeñas empresas se encuentran: la falta de recursos financieros; la falta de personal; la falta de tiempo y la falta de conocimientos tecnológicos (Medina et al., 2017). En esta idea redundan diversos estudios que establecen una relación directa entre el tamaño de la empresa y la innovación de ésta (Wanba, y Carter, 2014). Esta relación también ha sido estudiada con frecuencia en el sector cooperativo, señalando numerosos autores que la integración empresarial es uno de los factores determinantes para el fortalecimiento de las empresas (Arco, 1976; Martínez, 1990; Chaves, 1999; Mozas, 1999; Medina et al., 2017; entre otros). De esta forma, los retos de internacionalización, competitividad e innovación pasan por crecer en tamaño, siendo la integración una de las recomendaciones más extendidas (García, 2019; Hernández, 2019). Según estas argumentaciones es posible plantear la siguiente proposición en nuestro estudio de investigación:

\section{Proposición 5. El grado de integración empresarial, medido a través del número de cooperativas socias, favorece la innovación en la organiza- ción.}




\section{Metodología}

\subsection{Población}

Las organizaciones analizadas en este estudio acaparan un total de 449 empresas (444 cooperativas: 1 SAT; 3 SA; 1 grupo cooperativo), la mayoría de ellas cooperativas de primer grado, integrando a más de 165.000 socios individuales, que facturan más de 2 mil millones de euros y dan empleo a más de 2.500 personas. El grado de integración cooperativa en el sector oleícola supone el 50,16 por 100 de las cooperativas existentes y el 25 por 100 del total de almazaras (Mozas y Guzmán, 2017). Estas organizaciones se integran en torno a 35 sociedades cooperativas de segundo grado, dedicadas a la comercialización del aceite de oliva y constituyen nuestra población objeto de estudio. En España, las sociedades cooperativas de segundo grado son las mayores comercializadoras de aceite de oliva, integrando el 26 por 100 del total de la producción española (Mozas y Guzmán, 2017).

Para recabar los datos, se solicitó en primer lugar, la colaboración de Cooperativas Agroalimentarias de España, por ser la confederación que agrupa a la mayor parte del cooperativismo agrario español ${ }^{1}$. Dicha confederación realiza una encuesta estructurada anual en la que además del cooperativismo de primer grado también participan las cooperativas de segundo grado. Esta encuesta se realizó en el año 2018 y recoge los datos de las cooperativas correspondientes al año 2017. Por otra parte, en el año 2018 se llevó a cabo una encuesta telefónica estructurada dirigida a los responsables organizacionales de cada una de las 35 entidades (población total) que completó la información recogida en la base de datos de Cooperativa Agroalimentarias de España. Se obtuvieron los datos de 27 de ellas, lo que representaba una tasa de respuesta del $77,14 \%$.

\subsection{Método}

La técnica metodológica que ha sido utilizada en este estudio ha empleado la técnica Qualitative Comparative Analysis (QCA), empleando el planteamiento fuzzy sets (fsQCA), al objeto de establecer variables de corte tecnológico y organizacional que de forma conjunta se asocien a un mayor nivel de eficiencia. La técnica QCA, basada en el álgebra booleana, utiliza un lenguaje verbal, conceptual y matemático que la configura como un enfoque cualitativo y cuantitativo, aunando las principales ventajas de ambos (Ragin, 1987). De esta forma, aplicando QCA es posible analizar sistemáticamente un conjunto de casos, para determinar patrones causales en forma de relaciones de necesidad y de suficiencia, entre un conjunto de condiciones y un

1. Parte de los datos de este trabajo se han recopilado por parte de los investigadores en una encuesta realizada a la población objeto de estudio, pero otros, se solicitaron a Cooperativas Agro-alimentarias de España en el marco de los trabajos del Observatorio Socioeconómico del Cooperativismo Agroalimentario Español (OSCAE). Por tanto, este trabajo se ha realizado con la colaboración de Cooperativas Agro-alimentarias de España y el Ministerio de Agricultura, Pesca y Alimentación. 
resultado (Scheider y Wagemann, 2010). Este método tiene como ventaja, frente a una técnica de regresión, establecer relaciones entre subconjuntos de variables para explicar relaciones. Además, esta técnica permite trabajar con muestras de tamaño medio, no lo suficientemente grande como para aplicar métodos cuantitativos tradicionales (Ragin et al., 2003; Ragin y Rihoux, 2009).

Concretamente, en este estudio se ha hecho uso de la variante fuzzy sets (fsQCA), dado que solventa uno de los principales inconvenientes y críticas del planteamiento inicial denominado csQCA, como es su enfoque estrictamente dicotómico (Sehring et al., 2013).

La técnica QCA fue desarrollada para entornos de muestras o poblaciones pequeñas (Ragin, 1987), por lo que no supone un inconveniente para esta investigación, en la que el universo de estudio es pequeño. Para la correcta ejecución de esta técnica se siguen las fases recomendadas en la literatura, de calibración de variables, del análisis de necesidad y del análisis de suficiencia. Por ello, se procedió en primer lugar a la correspondiente calibración de variables. Acto seguido se realizó un análisis de necesidad de las puntuaciones de eficiencia sobre las distintas condiciones causales, para verificar que ninguno de los valores obtenidos supera el umbral recomendado en la literatura de 0,9, establecido por (Ragin, 2008), corroborándose esto.

La variable dependiente es el grado de innovación tecnológica. Esta variable ha sido creada considerando las diferentes TIC que utiliza la empresa en su desempeño empresarial. De este modo, se ha hecho un recuento del número de tecnologías de las que hace uso la empresa, considerándose las siguientes: intranet, sitio web, presencia en redes sociales, tienda electrónica, presencia en los mercados electrónicos y app. Por su parte, las variables causales utilizadas han sido: la formación en TIC de los recursos humanos (personal y máximo responsable); el grado de compromiso hacia la Responsabilidad Social Corporativa; el carácter exportador; la venta de oferta ecológica; y la integración empresarial.

Para el cálculo de la variable compromiso con la RSC hemos seguido las siguientes premisas. Por un lado, ninguna de las sociedades cooperativas analizadas presenta informes de RSC con los que poder medir su compromiso. Por otro lado, actualmente no existe una certificación única en RSC que acredita ésta, por lo que, las diversas certificaciones que existen en torno a los ámbitos o dimensiones de la RSC, son un referente informativo de los compromisos en RSC de las organizaciones (Chávez y Patraca, 2011). En consecuencia, la variable grado de compromiso hacia la Responsabilidad Social Corporativa se ha calculado a partir de las principales certificaciones de ámbito agroalimentario que desde la perspectiva social, económica y medioambiental comunican las cooperativas en su web. A modo de resumen, las principales certificaciones consideradas fueron:

- Dimensión social: certificaciones en Calidad y Seguridad alimentaria (ISO 9001; BRC, IFS, ISO 22000, GLOBAL GAP, ISO 22005, ...); y en materia Laboral, seguridad y salud del trabajo (ISO 45001, OHSAS 18001, SA 8000, existencia de planes de Igualdad y conciliación, modelo EFR (familiarmente responsable), SEDEX - SMETA Responsabilidad en la cadena de suministro, etc.).

- Dimensión medioambiental: certificaciones de la calidad medioambiental de la actividad (ISO 14001, Reglamento EMAS CE 1221/2009, residuo cero, FSC - PEFC, ISO 50001, 
certificación olivares vivos - SE0 BirdLife, producción integrada Andalucía, ISO 14064 huella de carbono de organizaciones (GHG Protocol y especificaciones sectoriales), ...); y certificaciones de calidad medioambiental del producto (GlobalEPD, huella de carbono del producto, huella hídrica, CAAE, denominación de origen, sello de "Calidad Certificada", otorgado por la Junta de Andalucía, certificado agricultura ecológica, ...).

- Dimensión económica: certificaciones de transparencia económica (informes financieros de la actividad) y de gestión (ISO 26000, modelo EFQR, memoria de sostenibilidad o RSC, SGE 21, SR10, Adhesión al Pacto Mundial, certificación FAIRTRADE, ...); así como otros indicadores (premios y reconocimientos, ISO 27001 de protección de datos, ISO 20000 de servicios TIC, ...).

En cómputo, todas estas variables se recogen en la tabla 1.

\section{Tabla 1. Variables consideradas para la técnica fsQ̨CA}

\begin{tabular}{ll} 
Variable de resultado & Descripción \\
\hline Innovación & Recuento de TIC que usa la organización $\quad$ Variable categórica'
\end{tabular}

\begin{tabular}{lll}
\hline Variables de condición & Descripción & \\
\hline Formación en TIC & $\begin{array}{l}\text { Formación en TIC del personal y responsable } \\
\text { de la organización }\end{array}$ & Variable categórica \\
\hline RSC & $\begin{array}{l}\text { Compromiso hacia la Responsabilidad Social } \\
\text { Corporativa }\end{array}$ & Variable continua $^{4}$ \\
\hline Internacionalización & La empresa exporta & Variable dicotómica2 \\
\hline Ecológico & Incluye productos ecológicos en su oferta & Variable dicotómica2 \\
\hline Integración & Número de sociedades que integra & Variable continua4 \\
\hline
\end{tabular}

1. Recuento de TIC de las que hace uso la empresa, considerándose las siguientes tecnologías: intranet, sitio web, presencia en redes sociales, tienda electrónica, presencia en los mercados electrónicos y app. Calibrada según Ragin \& Rihoux (2009).

2. Variable dicotómica (1: sí; $0:$ no).

3. Variable categórica de cuatro niveles (0,01: sin conocimientos; 0,33: nivel usuario; 0,67: nivel intermedio; 0,99: nivel avanzado). Calibrada según Ragin \& Rihoux (2009).

4. Las variables de tipo continuo han sido calibradas mediante el programa fsQCA 3.0.

Fuente: elaboración propia. 


\section{Resultados}

En una primera aproximación a los datos de este estudio, la tabla 2 recoge los valores promedio de las variables que han sido consideradas en el análisis.

\section{Tabla 2. Valores descriptivos}

\begin{tabular}{|c|c|}
\hline Variables & Valores medios de la muestra \\
\hline Innovación & $\begin{array}{l}\text { El } 29.63 \% \text { hace uso de intranet } \\
\text { El } 88.89 \% \text { dispone de sitio web } \\
\text { El } 40.74 \% \text { dispone de tienda virtual } \\
\text { El } 62.96 \% \text { usa las redes sociales virtuales } \\
\text { El } 11.11 \% \text { está en los mercados electrónicos } \\
\text { El } 7.41 \% \text { dispone de app }\end{array}$ \\
\hline Formación en TIC & $\begin{array}{l}\text { El } 59,26 \% \text { de los recursos humanos de la empresa tiene una } \\
\text { formación en TIC }\end{array}$ \\
\hline RSC & El 48,14\% de las sociedades presenta compromiso hacia la RSC \\
\hline Internacionalización & El $14.81 \%$ de las empresas no exporta \\
\hline Ecológico & El 29.62\% incluye productos ecológicos en su oferta \\
\hline Integración & Las sociedades integran de media unas 20 organizaciones \\
\hline
\end{tabular}

Fuente: elaboración propia.

Finalmente, los resultados del análisis de conjuntos difusos para empresas con un alto grado de innovación se presentan en la tabla 3, en el que se muestra la solución intermedia. Tal y como se utiliza en la literatura, los círculos negros (•) denotan la presencia de una condición, mientras que los círculos tachados $(\otimes)$ indican su ausencia (en el modelo no hay resultado de esta índole). La tabla de soluciones incluye valores de consistencia teórica de conjuntos para cada configuración, así como la solución general, con valor por encima del umbral recomendado en la literatura (>0.75) (Ragin, 2008). Las configuraciones causales han sido ordenadas de mayor a menor cobertura bruta. 


\section{Tabla 3. Resultados del análisis fsQ̨CA}

\begin{tabular}{lccc} 
SOLUCIONES & $\mathbf{1}$ & $\mathbf{2}$ & $\mathbf{3}$ \\
\hline RSC & $\bullet$ & $\bullet$ & $\bullet$ \\
\hline Formación en TIC & $\bullet$ & $\bullet$ & $\bullet$ \\
\hline Internacionalización & $\bullet$ & $\bullet$ & $\bullet$ \\
\hline Integración & & 0,469404 & 0,423510 \\
\hline Ecológico & & 0,574879 & 0,047504 \\
\hline Cobertura bruta & 0,198873 & 0,093398 & 0,956364 \\
\hline Cobertura única & 0,926671 & 0,888720 & \\
\hline Consistencia & $\mathbf{0 , 7 1 5 7 8 1}$ & & \\
\hline Cobertura del modelo: & $\mathbf{0 , 8 8 6 7 8 3}$ & & \\
\hline Consistencia del modelo: & & & \\
\hline
\end{tabular}

Fuente: elaboración propia.

Los resultados indican una cobertura global del modelo de 0,71 lo que sugiere que una proporción sustancial del resultado está cubierta por las cinco variables consideradas, con una consistencia global de 0,88 . La primera configuración explica por sí sola un 57,48 por ciento de las organizaciones que presentan una mayor innovación. Esta combinación de variables está integrada por las variables formación en TIC de los recursos humanos, el compromiso hacia la RSC y la internacionalización. La segunda configuración muestra que un 46,94 por ciento de las organizaciones que presentan mayor grado de innovación son aquellas que tienen compromiso hacia la RSC, cuentan con recursos humanos formados en TIC, exportan y tienen un mayor grado de integración. Por último, también cabe hacer mención a la tercera configuración causal, que abarca el 42,35 por ciento de los casos, referente a organizaciones que exportan, ofertan productos ecológicos, tienen un compromiso hacia la RSC y presentan una alta integración. En cómputo, todas las variables consideradas están presentes en el modelo con una relación positiva hacia la innovación. De este modo, se cumplen las proposiciones anteriormente descritas. 


\section{Conclusiones}

La literatura existente vincula directamente el desarrollo económico con la innovación y la inversión en Tecnologías de la Información y Comunicación (TIC). Así, el principal objetivo que nos marcábamos era comprobar cuáles son los factores estructurales, organizativos y comerciales que favorecen la innovación tecnológica en las cooperativas oleícolas de segundo grado en España. Los resultados obtenidos en este estudio permiten comprobar, mediante la utilización de la técnica fuzzy set Quality Comparative Analysis (fsQCA), que la inmersión de las empresas en las TIC se ve favorecida por los siguientes factores: la intensidad de integración cooperativa, medido por el número de cooperativas de primer grado u otras entidades que conforman la cooperativa de segundo grado; la formación en TIC de los empleados; el carácter exportador de la cooperativa, la oferta de productos ecológicos y el grado de compromiso de las cooperativas respecto a las acciones de Responsabilidad Social Corporativa (RSC).

La conclusión que extraemos es que lejos de considerar a la Responsabilidad Social Corporativa como una carga para las empresas e instituciones, debe analizarse e integrarse en las organizaciones como una oportunidad, puesto que este vínculo a través de ése círculo cerrado, sin duda, mejorará, no sólo la posición competitiva de la empresa, sino que ayudará a mejorar la situación de su entorno.

En el caso de las sociedades cooperativas, la RSC forma parte de su razón de ser (Mozas y Puentes, 2010; Castro, 2006; Carrasco, 2007) y aunque es poco comunicada al exterior (Mozas et al., 2014), aquellas que apuestan por la comunicación de sus compromisos con la RSC son también las que tienen una mayor orientación a las TIC. Esa vinculación con la Responsabilidad Social Corporativa, provocará que la cooperativa sea vista como una entidad comprometida con su entorno, mejorando su imagen de cara a sus potenciales clientes.

Esta línea de investigación centrada en el cooperativismo oleícola podría ser ampliada en futuras investigaciones que se centrasen en otros sectores de la Economía Social, con el objetivo de contrastar si los factores aquí marcados como determinantes para la innovación tecnológica de la empresa pueden ser establecidos de manera general en cualquier sector de actividad. Al respecto, sería muy interesante comprobar si los condicionantes diferenciadores del sector oleícola determinan que sean éstos unos factores clave para su avance en la innovación tecnológica.

\section{Bibliografía}

ANSOFF, H. I. (1976): La estrategia de la empresa; Universidad de Navarra: Pamplona, España.

ARC0, J. L. (1976): "El fenómeno de la concentración económica y su incidencia sobre las cooperativas", REVESCO, Revista de Estudios Cooperativos, 40, 3-41. 
ARRIETA, F.J. (2019): "Las cooperativas digitales como canales para el trabajo digital en un contexto de economía colaborativa", Boletín de la Asociación Internacional de Derecho Cooperativo, (54), 75-96. D0I: http://dx.doi.org/10.18543/baidc-54-2019pp75-96.

BERNAL, E., MOZAS, A., FERNÁNDEZ, D. \& MEDINA, M.J. (2017): “Explanatory factors for efficiency in the use of social networking sites. The case of organic food products", Psychology \& Marketing, 34(12), 1119-1126.

CARRASCO, I. (2007): “Corporate social responsibility, values and cooperation", International Advances in Economic Research, 13(4), 454-460.

CASTRO, M. (2006): “Las empresas de economía social y la responsabilidad social corporativa”, Papeles de Economía Española, 108, 92-105.

CEGARRA, J. G., REVERTE, C., GÓMEZ, E., \& WENSLEY, A. K. (2016): "Linking social and economic responsibilities with financial performance: The role of innovation", European Management Journal, 34(5), 530-539.

CHAVES, R. (1999): “Grupos empresariales de la economía social: un análisis desde la experiencia española", en: Grupos empresariales de la Economía Social en España; Barea, J., Juliá, J.F., Monzón, J.L., Eds; CIRIEC-España: Valencia, España, 67-98.

CHÁVEZ, C. y PATRACA, V. (2011): “El comercio justo y la responsabilidad social empresarial. Reflexiones desde los sistemas de certificación autónoma", Argumentos (Méx.), 24 (65), 229259.

CONSEJO ECONÓMICO Y SOCIAL DE LA PROVINCIA DE JAÉN (CES) (2019): Memoria 2018 sobre la situación socioeconómica y laboral de la provincia de Jaén, Diputación Provincial de Jaén, Jaén.

CONSEJO OLEÍCOLA INTERNACIONAL, COI (2019a): "Production Olive Oils in UE" http://www.internationaloliveoil.org/estaticos/view/131-world-olive-oil-figures, revisado en junio de 2019.

CONSEJO OLEÍCOLA INTERNACIONAL, COI (2019b): "Production Olive Oils" http://www.internationaloliveoil.org/estaticos/view/131-world-olive-oil-figures, revisado en junio de 2019.

COOPERATIVAS AGROALIMENTARIAS DE ESPAÑA (2017): El cooperativismo en cifras. Disponible en: http://www.agro-alimentarias.coop/cooperativismo_en_cifras, revisado en abril de 2017.

EBA, E. y GONZALO E. (2019): "La formación en los valores cooperativos como elemento decisivo para los nuevos trabajos que crean las cooperativas del siglo XXI", Boletín de la Asociación Internacional de Derecho Cooperativo, 54, 97-114. DOI: http://dx.doi.org/10.18543/baidc-542019pp97-114. 
FELDMAN, M.P. (1993): "An examination of the geography of innovation", Industrial and Corporate Change, 2(3), 451-470.

FERNÁNDEZ, D., BERNAL, E., MOZAS, A. \& MEDINA, M.J. (2019): “The importance of websites for organic agri-food producers", Economic Research-Ekonomska Istraživanja, 1-14.

FIERRO, E., MERCADO, P. y CERNAS, D.A. (2013): "El efecto de la cultura centrada en el conocimiento y la interacción social en la innovación organizativa. El efecto mediador de la gestión del conocimiento", Esic Market Economics and Business Journal, 44(2), 87-108.

GARCÍA, A., ESPASANDÍN, F. \& BORRA, C. (2008): "Innovative behaviour in social economy: The Andalusian case", Scientific Annals of the "Alexandru Ioan Cuza", University of lasi: Economic Sciences Series, 55, 255-265.

GARCÍA, E. (2019): "Las sociedades cooperativas agroalimentarias ante la aplicación de las normas de competencia", CIRIEC-España, Revista Jurídica de Economía Social y Cooperativa, 35. http://ciriec-revistajuridica.es/wp-content/uploads/comen35-12.pdf.

GUERRAS, L.A. y NAVAS, J.E. (2015): La Dirección estratégica de la empresa: teoría y aplicaciones; Thomson/Cívitas: Pamplona, España.

HERNÁNDEZ, D. (2019): "El fomento de la integración de las cooperativas agroalimentarias andaluzas a través del reconocimiento como entidad asociativa prioritaria agroalimentaria", CIRIEC-España, Revista Jurídica de Economía Social y Cooperativa, 34.

http://ciriec-revistajuridica.es/wp-content/uploads/comen34-08.pdf

HOLLENSTEIN, H. (2004): "Determinants of the adoption of information and communication technologies (ICT). An empirical analysis based on firm level data for the swiss business sector", Structural Change and Economic Dynamics, 15 (3), 315-342.

JULIÁ, J.F. (1993): “La economía social y el cooperativismo agrario. Sus nuevas estrategias empresariales", CIRIEC-España. Revista de Economía Pública, Social y Cooperativa, 15, 43-68.

KIM, M.K. \& JEE, K.Y. (2007): "Factors influencing strategic use of information technology and its impact on business performance of SMEs", ETRI journal, 29 (4), 497-506.

LEE, H.J. \& YUN, Z.S. (2015): “Consumers' perceptions of organic food attributes and cognitive and affective attitudes as determinants of their purchase intentions toward organic food", Food Quality and Preference, 39, 259-267.

LEVIE, J. \& AUTIO, E. (2011): "Regulatory burden, rule of law, and entry of strategic entrepreneurs: An international panel study", Journal of Management Studies, 48 (6), 1392-1419.

LÓPEZ, A., VALLE, R. \& HERRERO, I. (2006): "The contribution of core employees to organizational capabilities and efficiency", Human Resource Management, 45(1), 81-109. 
MACGREGOR, P. \& FONTRODONA, J. (2008): Exploring the Fit between CSR and Innovation, IESE Business School, Working Paper No. 759.

Available at: http://dx.doi.org/10.2139/ssrn.1269334

MANYIKA, J. \& LUND, S. (2016): Globalization for the little guy, McKinsey Global Institute report: Madrid, España.

MARTíNEZ, A. (1990): Análisis de la integración cooperativa, Universidad Deusto, Bilbao, España.

MEDINA, M.J., BERNAL, E., FERNÁNDEZ, D. (2017): El impacto en el medio ambiente del sector oleícola mediante su avance comercial online, Instituto de Estudio Giennenses, Diputación de Jaén: Jaén.

MEDINA, M.J., BERNAL, E., MOZAS, A., MORAL, E. \& FERNÁNDEZ, D. (2016): "Efficiency of organic farming companies that operate in an online environment”, Custos e @gronegocio, 11(4), 264-289.

MÉNDEZ, R. (1998): "Innovación tecnológica y reorganización del espacio industrial: Una propuesta metodológica", EURE (Santiago), 24(73), 31-54.

MORENO, A.S. y SEMPERE, S.P. (2019): "Análisis de las plataformas de crowdfunding social en el estado español: un estudio de casos", REVESCO, Revista de Estudios Cooperativos, 130, 149-175.

MOZAS, A. (1999): Organización y gestión de las almazaras cooperativas: un estudio empírico; Junta de Andalucía, Consejería de Trabajo e Industria, Dirección General de Cooperativas: Sevilla, España.

MOZAS, A. (2019a): "El cooperativismo y la economía social en la implementación de los ODS", Noticias de la Economía Pública, Social y Cooperativa, 61, 36-38.

http://ciriec.es/wp-content/uploads/2019/11/Noticias_CIDEC61_web.pdf

MOZAS, A. (2019b): Contribución de las Cooperativas Agrarias al cumplimiento de los Objetivos de Desarrollo sostenible. Especial referencia al sector oleícola, CIRIEC-España, Valencia.

MOZAS, A. y GUZMÁN, A. (2017): "La evolución del cooperativismo oleícola: integración y cooperación", en: Economía y comercialización de los aceites de oliva. Factores y perspectivas para el liderazgo español del mercado global, Gómez-Limón, J. A., Parras, M., Eds., CAJAMAR Caja Rural: Almería, España, 107-130.

MOZAS, A. y PUENTES, R. (2010): “La responsabilidad social corporativa y su paralelismo con las sociedades cooperativas", REVESCO, Revista de Estudios Cooperativos, 103,1-22.

MOZAS, A., BERNAL, E. \& PUENTES, R. (2014): "Spanish second-tier cooperative societies: An analysis of their e-corporate social responsibility", Journal of Rural Cooperation, 42 (1), 30-59. 
PEDERSEN, E.R.G., GWOZDZ, W. \& HVASS, K.K. (2018): “Exploring the relationship between business model innovation, corporate sustainability, and organisational values within the fashion industry", Journal of Business Ethics, 149(2), 267-284.

PORTER, M.E. (1985): Competitive Advantage: Creating and Sustaining Superior Performance, Free Press, New York, NY.

PORTER, M.E. (1990): The competitive advantage of nations, McMillan, New York. La ventaja competitiva de las naciones, Plaza and Janes Editors, 1991, versión manejada.

PRIETO, A.V. \& GUZMÁN, D.C.C. (2019): “Efecto de las cooperativas exportadoras de café en el crecimiento de la economía solidaria en Colombia", REVESCO, Revista de Estudios Cooperativos, 130, 213-234.

QURRATU'AINI, H. \& HAPSARI, A.Y. (2019): “Interests Influence of Digital Marketing Product Sales in Exports by SMEs in Bandung", Global Business and Management Research, 11(1), 217-225.

RAGIN, C.C. (1987): The comparative method: moving beyond qualitative and quantitative strategies, University of California Press, Berkeley.

RAGIN, C.C. (2008): Redesigning social inquiry: fuzzy sets and beyond, University of Chicago Press: Chicaga, USA.

RAGIN, C.C. \& RIHOUX, B. (2009): Configurational comparative methods: Qualitative comparative analysis (QCA) and related techniques, Sage: California, USA.

RAGIN, C.C. \& RIHOUX, B. (2004): "Qualitative comparative analysis (QCA): state of the art and prospects", Qualitative Methods, 2(2), 3-13.

RAGIN, C.C., SHULMAN, D., WEINBERG, A. \& GRAN, B. (2003): “Complexity, generality and qualitative comparative analysis", Field Methods, 15(4), 323-340.

RATAJCZAK, P. \& SZUTOWSKI, D. (2016): "Exploring the relationship between CSR and innovation", Sustainability Accounting, Management and Policy Journal. 7(2), 295-318.

REGLAMENTO DEL PARLAMENTO EUROPEO Y DEL CONSEJO por el que se establece el programa Europa Digital para el período 2021-2027, (2018/0227 COD) visto en:

https://eur-lex.europa.eu/legal-content/ES/TXT/HTML/?uri=CELEX:52018PC0434\&from=EN

REXHEPI, G., KURTISHI, S. \& BEXHETI, G. (2013): “Corporate social responsibility (CSR) and innovation the drivers of business growth", Procedia-Social and Behavioral Sciences, 75(3), 532-541.

ROTHWELL, J. (1983): "Innovation and firm size: a case for dynamic complementarity; or small really beautiful?", Journal of General Management, 8(3), 5-25. 
SCHNEIDER, C.Q. \& WAGEMANN, C. (2010): "Standards of good practice in qualitative comparative analysis (QCA) and fuzzy-sets", Comparative Sociology, 9(3), 397-418.

SCHUMPETER, J.A. (1934): Theory of Economic Development: An Inquiry into Profits, Capital, Credit, Interest, and the Business Cycle, Cambridge, MA: Harvard University Press.

SEHRING, J., KORHONEN, K. \& BROCKHAUS, M. (2013): Qualitative Comparative Analysis (QCA): An application to compare national REDD+ policy processes. In CIFOR: Indonesia.

SHER, P.J. \& YANG, P.Y. (2005): "The Effects of Innovative Capabilities and R\&D Clustering on Firm Performance: The Evidence of Taiwan's Semiconductor Industry", Technovation, 25, 33-43.

TETHER, B.S. (2005): "Do services innovate (differently)? Insights from the European innobarometer survey", Industry and Innovation, 12(2), 153-184.

VEGA, M., TORRES, F.J. Y PARRAS, M. (2018): “Key Determinants of Organic Food Consumption: The Case of Olive Oil in Spain", HortScience, 53(8), 1172-1178.

VILAR, J. y CÁRDENAS, J. R. (2016): El sector internacional de elaboración de aceite de oliva. Un estudio descriptivo de los 56 países productores, GEA Centro Internacional de Excelencia para Aceite de Oliva, Jaén.

VILLAESCUSA, E. (2019): "Los ODS son el escenario natural de las cooperativas", Noticias de la Economía Pública, Social y Cooperativa, 61, 43-45.

http://ciriec.es/wp-content/uploads/2019/11/Noticias_CIDEC61_web.pdf

WAMBA, S.F. \& CARTER, L. (2014): "Social Media Tools Adoption and Use by SMEs: An Empirical Study", Journal of Organizational and End User Computing, 26(2), 1-17. 